PROCEEDINGS OF THE

AMERICAN MATHEMATICAL SOCIETY

Volume 130, Number 4, Pages 1031-1034

S 0002-9939(01)06187-1

Article electronically published on October 12, 2001

\title{
OVERSAMPLING AND PRESERVATION OF TIGHTNESS IN AFFINE FRAMES
}

\author{
RAQUEL G. CATALÁN
}

(Communicated by David R. Larson)

\begin{abstract}
The problem of how an oversampling of translations affects the bounds of an affine frame has been proposed by Chui and Shi. In particular, they proved that tightness is preserved if the oversampling factor is coprime with the dilation factor. In this paper we study, in the dyadic dilation case, oversampling of translation by factors which do not satisfy the above condition, and prove that tightness is preserved only in the case of affine frames generated by wavelets having frequency support with very particular properties.
\end{abstract}

\section{INTRODUCTION}

Let $(\mathbb{H},\langle\cdot, \cdot\rangle)$ be a separable Hilbert space. Let $I$ be a countable set of indices. A sequence $\left\{x_{i}\right\}_{i \in I}$ in $\mathbb{H}$ is a frame for $\mathbb{H}$ if there exist constants $0<A \leq B$ such that for every $y \in \mathbb{H}$ we have

$$
A\|y\|_{\mathbb{H}}^{2} \leq \sum_{i \in I}\left|\left\langle x_{i}, y\right\rangle\right|^{2} \leq B\|y\|_{\mathbb{H}}^{2} .
$$

If we can choose $A=B$, the frame is said to be tight, with constant $A$.

The main advantage of frames that are not Riesz bases is the combination of the stability of the system with the possibility of redundancy, which can be used effectively in many applications, such as removing uncorrelated noise.

For $\psi \in L^{2}(\mathbb{R})$, let

$$
\Psi\left(a_{0}, b_{0}\right)=\left\{\psi_{j, n}=a_{0}^{\frac{j}{2}} \psi\left(a_{0}^{j} \cdot-n b_{0}\right) ; j, n \in \mathbb{Z}\right\} .
$$

In this paper we will mainly restrict our attention to the particular case $a_{0}=2$, $b_{0}=1$. The theorem in 1 implies that if $\Psi\left(2, b_{0}\right)$ is a tight frame, then the oversampled system $\Psi\left(2, \frac{b_{0}}{k}\right)$ is also a tight frame when $k$ is any odd integer. As observed there, this result is sharp, in the sense that if $k$ is even, the oversampled system $\Psi\left(2, \frac{1}{k}\right)$ is not necessarily a tight frame even when $\Psi(2,1)$ is: the Haar wavelet provides an immediate counterexample.

Our aim is to study for which $\psi$ it is true that if $\Psi(2,1)$ is a tight frame, then the oversampled system $\Psi\left(2, \frac{1}{n 2^{r}}\right)$ is a tight frame. It turns out that conditions on

Received by the editors September 3, 1999 and, in revised form, September 29, 2000.

2000 Mathematics Subject Classification. Primary 42C40.

Key words and phrases. Wavelets, frames, tight frames.

This work was partially supported by the Spanish DGES PB97-1013, and originated during a stay at the Politecnico di Torino with the European TMR network on "Applications of the wavelet element method to boundary value problems". 
the support of $\hat{\psi}$ are the key for solving this problem. We introduce the following definition:

Definition 1.1. A function $f \in L^{2}(\mathbb{R})$ is band limited up to congruences with band width $B$ (we will say that $f$ is $B-B L c$ ) if there exist bounded intervals $\left\{I_{k}, k \in \mathbb{Z}\right\}$ such that the translations $J_{k}=I_{k}-k B$ are disjoint, $J_{k} \subset[-B, B]$ and $\operatorname{supp} \hat{f} \subset \bigcup_{k \in \mathbb{Z}} I_{k}$.

\section{RESULts}

We will denote by $\mathcal{F}_{k}$ the set of wavelets $\psi$ such that the associated family $\Psi\left(2, \frac{1}{k}\right)$ is a tight frame for $L^{2}(\mathbb{R})$ with constant $k$. We recall the following characterization of tight frame wavelets [11, 5, 7]:

Theorem 2.1. Let $\psi \in L^{2}(\mathbb{R})$. Then $\psi \in \mathcal{F}_{k}$ if and only if the following two conditions hold almost everywhere:

i) $\sum_{j \in \mathbb{Z}}\left|\hat{\psi}\left(2^{j} \xi\right)\right|^{2}=k$,

ii) $t_{q}^{k}(\xi):=\sum_{j=0}^{\infty} \hat{\psi}\left(2^{j} \xi\right) \overline{\hat{\psi}\left(2^{j}(\xi-2 \pi k q)\right)}=0 \forall q \in 2 \mathbb{Z}+1$.

These two equations will be the main tool in our investigation. They will allow us to prove the following results.

Theorem 2.2. For any $\psi \in \mathcal{F}_{1}$ the following three statements are equivalent:
a) $\psi \in \bigcap_{n \in \mathbb{N}} \mathcal{F}_{2 n}$,
b) $\psi \in \bigcap_{r \in \mathbb{N}} \mathcal{F}_{2^{r}}$,
c) $\psi$ is $2 \pi-B L c$.

Corollary 2.3. A wavelet $\psi \in \mathcal{F}_{1}$ is in $\mathcal{F}_{n}$ for all $n \in \mathbb{N}$ if, and only if, it is $2 \pi-B L c$

For more general oversampling factors, we obtain the following

Theorem 2.4. Let $n_{0}, r_{0} \in \mathbb{N}, \psi \in \mathcal{F}_{1}$. Then $\psi \in \bigcap_{r=r_{0}}^{\infty} \mathcal{F}_{2^{r} n_{0}}$ if, and only if, it is $2 \pi 2^{r_{0}} n_{0}-B L c$

Corollary 2.5. Let $n_{0} \in \mathbb{N}, \psi \in \mathcal{F}_{1}$. If $\psi \in \mathcal{F}_{m}$ for all $m \geq n_{0}$, then $\psi$ is $2 \pi \bar{n}_{0}$ $B L c$, where $\bar{n}_{0}=n_{0}$ if $n_{0}$ is even and $\bar{n}_{0}=n_{0}+1$ otherwise.

Our last result deals with oversampling of wavelets having compactly supported Fourier transform:

Theorem 2.6. Let $\psi$ be a band limited wavelet and $a_{0}>1, b_{0}>0$ be such that $\Psi\left(a_{0}, b_{0}\right)$ is a frame with constants $A, B$. Then there exists $\lambda_{0}>0$ so that for any $\lambda \geq \lambda_{0}$ the family $\Psi\left(a_{0}, \frac{b_{0}}{\lambda}\right)$ is also a frame, with admissible bounds $\lambda A, \lambda B$. 


\section{Proofs}

Proof of Theorem 2.2. Suppose $\Psi(2,1)$ is a tight frame. We study first the conditions that guarantee that $\Psi\left(2, \frac{1}{2}\right)$ is a tight frame. By Theorem 2.1 we need to impose, for any odd $q$,

$$
\begin{aligned}
0=t_{q}^{2}(\xi) & =\sum_{j=0}^{\infty} \hat{\psi}\left(2^{j} \xi\right) \overline{\hat{\psi}\left(2^{j}(\xi-2 \pi 2 q)\right)} \\
& =-\hat{\psi}\left(\frac{\xi}{2}\right)+\sum_{j=0}^{\infty} \hat{\psi}\left(2^{j} \frac{\xi}{2}\right) \overline{\hat{\psi}\left(2^{j}\left(\frac{\xi}{2}-2 \pi q\right)\right)} .
\end{aligned}
$$

The last series sums to 0 a.e. By Theorem 2.1 and the assumption that $\Psi(2,1)$ is a tight frame, so $\Psi\left(2, \frac{1}{2}\right)$ is a tight frame if and only if

$$
\hat{\psi}(\cdot) \overline{\hat{\psi}(\cdot-2 \pi q)}=0
$$

for every $q \in 2 \mathbb{Z}+1$. By induction one deduces the conditions for $\Psi\left(2, \frac{1}{2^{r}}\right)$ to be a tight frame. For a fixed $h \in \mathbb{N}, \psi$ is $2 h \pi$-BLc if and only if, for all $n \in \mathbb{Z}$, $\hat{\psi}(\cdot) \overline{\hat{\psi}(\cdot-2 \pi h n)}=0$. The thesis and the various corollaries then follow by writing an arbitrary oversampling factor $k$ in the form $2^{r} n$, for some $r \in \mathbb{N}$, and odd integer $n$.

Proof of Theorem 2.6. Since the family $\Psi(a, b)$ is a frame for $L^{2}(\mathbb{R})$ with constants $A, B$, then ([3])

$$
A b \leq \sum_{j \in \mathbb{Z}}\left|\hat{\psi}\left(a^{-j} \xi\right)\right|^{2} \leq B b \quad \text { a.e. }
$$

Let $f \in L^{2}(\mathbb{R})$, and take $\lambda \geq \lambda_{0}>0$ such that $\operatorname{supp} \hat{\psi} \subset\left[-\frac{\pi \lambda_{0}}{b}, \frac{\pi \lambda_{0}}{b}\right]$. Then, by Plancherel's theorem

$$
\begin{aligned}
\left\langle f, \psi_{j, n}^{\lambda}\right\rangle & =\frac{1}{2 \pi}\left\langle\hat{f}, \psi_{j, n}^{\hat{\lambda}}\right\rangle \\
& =\frac{1}{2 \pi} a^{\frac{j}{2}} \sqrt{\frac{\lambda}{b}} \int_{-\frac{\pi \lambda}{b}}^{\frac{\pi \lambda}{b}} \hat{f}\left(a^{j} \xi\right) \overline{\hat{\psi}(\xi)} \sqrt{\frac{b}{\lambda}} e^{i \xi n \frac{b}{\lambda}} d \xi .
\end{aligned}
$$

Since $\left\{\sqrt{\frac{b}{\lambda}} e^{-i n \frac{b}{\lambda} \cdot} ; n \in \mathbb{Z}\right\}$ is an orthonormal basis in $L^{2}\left(\left[-\frac{\pi \lambda}{b}, \frac{\pi \lambda}{b}\right]\right)$, the summation in $n$ gives $\frac{1}{2 \pi} \frac{\lambda}{b} \int_{\mathbb{R}}|\hat{f}(\xi)|^{2}\left|\hat{\psi}\left(a^{-j} \xi\right)\right|^{2} d \xi$ and the sum in $j$ then yields the condition of frame with the announced bounds.

\section{ACKNOWLEDGMENT}

The author thanks Professor Guido Weiss for some useful observations that are included in this article.

\section{REFERENCES}

[1] C.K.Chui-X.Shi, Bessel sequences and Affine frames, Applied and Computational Harmonic Analysis, n. 1, pp. 29-49 (1993). MR 95b:42028

[2] C.K.Chui-X.Shi, $n \times$ oversampling preserves any tight affine frame for odd $n$, Proceedings of the AMS, Vol. 121, n.2, pp. 511-517. (1994). MR 94h:42052

[3] I.Daubechies, Ten Lectures on Wavelets, CBMS, (1992). MR 93e:42045 
[4] - The wavelet transform, time-frequency localization, and signal analysis, IEEE Trans. Information Theory, Vol. 36, pp. 961-1005 (1990). MR 91e:42038

[5] G.Gripenberg, A necessary and sufficient condition for the existence of a father wavelet, Studia Math. 114(3), pp. 207-226 (1995). MR 96d:42049

[6] C.Heil, D.Walnut, Continous and discrete wavelet transform, SIAM Review, 31, pp. 628-666 (1989). MR 91c:42032

[7] E.Hernandez-G.Weiss, A first course on wavelets, CRC Press (1996). MR 97i:42015

[8] Y.Meyer, Ondelettes et operateurs, II, Hermann (1990). MR 93i:42003

[9] D.Walnut, Continuity properties of the Gabor frame operator, J. Math.Analysis. Appl. 165, pp. 479-504 (1992). MR 93f:42059

[10] Weil-Heisenberg wavelet expansion: existence and stability in weighted spaces, $\mathrm{Ph}$. D. Thesis. University of Maryland (1989).

[11] X.Wang, The study of wavelets from the properties of their Fourier Transforms, Ph.D.Thesis, Washington University in St. Louis (1995).

[12] R. Young, An Introduction to Nonharmonic Fourier Series, Academic Press (1980). MR 81m:42027

Departamento de Matemática e Informática, Universidad Pública de Navarra, 31006 , PAMPlOna, Spain

E-mail address: raquel.garcia@unavarra.es 